\section{(1) \\ CrossMark}

\title{
Subglottic secretion drainage for preventing ventilator-associated pneumonia: an overview of systematic reviews and an updated meta-analysis
}

\author{
Diana P. Pozuelo-Carrascosa (10 ${ }^{1,2}$, Ángel Herráiz-Adillo $\mathbb{1}^{3}$, Celia Alvarez-Bueno ${ }^{1}$, \\ Jose Manuel Añón ${ }^{4}$, Vicente Martínez-Vizcaíno ${ }^{1,5}$ and Iván Cavero-Redondo ${ }^{1}$
}

\begin{abstract}
Affiliations: ${ }^{1}$ Health and Social Care Research Center, Universidad de Castilla-La Mancha, Cuenca, Spain. ${ }^{2}$ Faculty of Physiotherapy and Nursing, Universidad de Castilla-La Mancha, Toledo, Spain. ${ }^{3}$ Dept of Primary Care, Health Service of Castilla-La Mancha (SESCAM). Tragacete, Cuenca, Spain. ${ }^{4}$ Intensive Care Unit, Hospital Universitario La Paz-Carlos III, IdiPAZ, CIBER de Enfermedades Respiratorias, Instituto de Salud Carlos III, Madrid, España. ${ }^{5}$ Universidad Autónoma de Chile, Faculty of Health Sciences, Madrid, Spain.
\end{abstract}

Correspondence: Ángel Herráiz Adillo, Health Service of Castilla-La Mancha (SESCAM), Dept of Primary Care, Tragacete, Plaza de la Constitución 7, 16150 Cuenca, Spain. E-mail: ahadilloQhotmail.com

@ERSpublications

Subglottic secretion drainage is an effective measure to reduce mortality and VAP incidence, despite not improving the duration of mechanical ventilation or length of stay in ICU and/or hospital. http://bit.ly/2PeJLR1

Cite this article as: Pozuelo-Carrascosa DP, Herráiz-Adillo Á, Alvarez-Bueno C, et al. Subglottic secretion drainage for preventing ventilator-associated pneumonia: an overview of systematic reviews and an updated meta-analysis. Eur Respir Rev 2020; 29: 190107 [https://doi.org/10.1183/16000617.0107-2019].

ABSTRACT Although several guidelines recommend subglottic secretion drainage as a strategy for prevention of ventilator-associated pneumonia (VAP), its use is not widespread. With the aim to assess the effectiveness of subglottic secretion drainage for preventing VAP and to improve other outcomes such as mortality, duration of mechanical ventilation and length of stay in the intensive care unit (ICU) or hospital, an electronic search of the Cochrane Library, MEDLINE, Web of Science and Embase was undertaken. Nine systematic reviews with meta-analysis (in the overview of reviews) and 20 randomised controlled trials (in the updated meta-analysis) were included. In the overview of reviews, all systematic reviews with meta-analysis included found a positive effect of subglottic secretion drainage in the reduction of incidence of VAP. In the updated meta-analysis, subglottic secretion drainage significantly reduced VAP incidence (risk ratio (RR) $0.56,95 \%$ CI $0.48-0.63 ; \mathrm{I}^{2}=0 \%, \mathrm{p}=0.841$ ) and mortality (RR 0.88 , 95\% CI $0.80-0.97 ; \mathrm{I}^{2}=0 \%, \mathrm{p}=0.888$ ).

This is the first study that has found a decrease of mortality associated with the use of subglottic secretion drainage. In addition, subglottic secretion drainage is an effective measure to reduce VAP incidence, despite not improving the duration of mechanical ventilation and ICU and/or hospital length of stay.

Since publication of this article, it has been found that there is an error in the analysis presented in figure 3 . This is indicated in the correction notice published in volume 31, issue 163 of the European Respiratory Review, and fully clarified by the authors in their correspondence to the editor published in the same issue (https://doi.org/10.1183/16000617.0013-2022)

This article has supplementary material available from err.ersjournals.com

Provenance: Submitted article, peer reviewed

Received: 15 Aug 2019 | Accepted after revision: 21 Oct 2019

Copyright $\odot$ ERS 2020. This article is open access and distributed under the terms of the Creative Commons Attribution Non-Commercial Licence 4.0. 


\section{Introduction}

Ventilator-associated pneumonia (VAP) is a hospital-acquired pneumonia developed in intubated patients receiving mechanical ventilation for $\geqslant 48 \mathrm{~h}$ [1]. VAP is among the highest incidence hospital-acquired infections in intensive care units (ICU) affecting one-third of patients with mechanical ventilation and having an attributable mortality of $4.6-13 \%[2,3]$. The high incidence and mortality attributable to VAP are associated with increased resource utilisation burden generating a high economic cost to healthcare systems. Thus, cost-effective interventions that minimise the incidence of this adverse event are needed [4].

The necessary use of the endotracheal tube is one of the main risk factors for the development of VAP. It interferes with the normal protective upper airway reflexes, reduces effective coughing, causes irritation of the respiratory mucosa, increases the amount of mucus and promotes microaspiration of contaminated oropharyngeal secretions [5]. This microaspiration is the main mechanism for the entry of bacteria into the lower airway $[6,7]$, which could lead to VAP, depending on the quantity and virulence of aspirated bacteria and the patient's defence mechanisms [8].

Subglottic secretion drainage (SSD) has been studied extensively as a strategy for VAP prevention in such a way that $>20$ randomised controlled trials (RCTs) and several meta-analyses have assessed the effectiveness of this technique to reduce incidence of VAP. Nevertheless, although several guidelines recommend its use, the use of SSD is not widespread, probably due to the weakness of the quality of evidence [9-12].

For these reasons, the aim of this study was to synthesise the available evidence providing an overview of systematic reviews about the effectiveness of SSD to reduce VAP, length of stay in ICU and/or hospital, duration of mechanical ventilation and mortality. Additionally, an updated meta-analysis was conducted, which aimed to provide updated evidence about this topic.

\section{Methods}

This overview of systematic reviews and the updated meta-analysis were registered in the International Prospective Register of Systematic Reviews (PROSPERO) database (CRD42019123699). The updated meta-analysis was performed according to the Preferred Reporting Items for Systematic Reviews and Meta-analysis (PRISMA) statement [13], and the recommendations of the Cochrane Handbook for Systematic Reviews of Interventions [14] were followed.

\section{Data sources and search methods}

An electronic search of the Cochrane Library, MEDLINE (via PubMed), Web of Science and Embase (via Scopus) was undertaken, from inception to July 17, 2019, to identify systematic reviews with meta-analysis and RCTs that aimed to assess the effectiveness of SSD to prevent VAP.

The search strategy was "ventilator-associated pneumonia" AND ("subglottic secretion" OR "subglottic aspiration" OR "subglottic drainage" OR "subglottic suctioning" OR "endotracheal intubation" OR “endotracheal tube" OR "endotracheal cuff”) AND ("meta-analysis" OR "systematic review" OR "randomised controlled trial"). There were no date or language restrictions. In addition, the reference list of published full-text articles and systematic reviews were manually scanned for relevant studies (meta-analyses and RCTs).

\section{Selection criteria and data extraction}

The inclusion criteria for the overview of systematic reviews with meta-analysis were 1) population: adult patients admitted to ICUs; 2) type of study: systematic reviews with meta-analysis of RCTs; 3) type of intervention: use of endotracheal tubes that allow SSD for VAP prevention compared with the standard endotracheal tube or non-SSD control group; 4) primary outcome: VAP incidence; and 5) secondary outcomes: ICU and/or hospital mortality, duration of mechanical ventilation, ICU and/or hospital length of stay and time to VAP.

Additionally, the identified independent RCTs that aimed to assess the effectiveness of SSD to prevent VAP in adults admitted to ICUs were selected and reviewed for an updated meta-analysis. Studies in children or paediatric ICUs and studies with inconsistent or insufficient data were excluded. Abstract publications were also excluded.

Two independent reviewers (DPP-C and AH-A) conducted both the selection of studies and data extraction. Duplicate studies and irrelevant titles were removed. Disagreements were solved by discussion, and if disagreement persisted, a third reviewer solved the conflict (VM-V). Reviewers were not blinded to authors, journals or institutions.

Data from the included systematic reviews with meta-analysis were extracted through a standard data extraction form; the original studies were used when specific data were missing. The following data from each included systematic review were extracted: 1) first author's name; 2) year of publication; 3) number of 
included RCTs and participants; 4) study aim; 5) eligibility criteria (details of the included participants and details of the intervention studied); 6) data for the risk ratio (RR) for dichotomous outcomes (VAP incidence and mortality) and mean differences for continuous outcomes (duration of mechanical ventilation, ICU and/or hospital length of stay and time-to-VAP); and 7) any additional methodological information of potential importance, such as the assessment of methodological quality, risk of bias, limitations and quality of evidence.

Additionally, each RCT was reviewed for necessary data; when some data were not available, each systematic review with meta-analysis in which that study was included was also reviewed to acquire any missing data. The following data were extracted from each included article: 1) first author's name; 2) year of publication; 3) study name and study aim; 4) participant characteristics (number, age); 5) intervention characteristics (SSD method, co-interventions in the intervention group, VAP prevention bundle); and 6) VAP incidence, ICU and/or hospital length of stay, duration of mechanical ventilation, time to VAP and mortality both in the control and intervention groups.

\section{Quality assessment}

Two authors (DPP-C and AH-A) independently rated the methodological quality and the quality of evidence of each outcome of the systematic reviews with meta-analysis, and the risk of bias of the RCTs included.

\section{Overview of reviews \\ Methodological quality of included reviews}

The Assessment of Multiple Systematic Reviews (AMSTAR-2) tool was used to assess the methodological quality of each included review [15]. This tool consists of 16 items in total with simple response categories. These items include, among others, the presence of a protocol, comprehensiveness of the literature search and assessment of the risk of bias of the individual studies included in the systematic review.

\section{Quality of evidence}

The Grading of Recommendations, Assessment, Development and Evaluations (GRADE) tool was used to assess the quality of the evidence related to the key outcomes when the reviews did not provide a GRADE assessment [16]. This system uses the following criteria to assign a quality level to a body of evidence [14]: 1) high (randomised trials or double-upgraded observational studies); 2) moderate (downgraded randomised trials or upgraded observational studies); 3) low (double-downgraded randomised trials or observational studies); 4) very low (triple-downgraded randomised trials, downgraded observational studies or case series/case reports).

Additionally, some factors which can increase and/or decrease the quality of evidence were considered: 1) limitations of the studies, such as the likelihood of bias (downgraded once if $<75 \%$ of the included studies were at low risk of bias); 2) inconsistency including unexplained heterogeneity or inconsistency of results (downgraded once when the $\mathrm{I}^{2}$ statistic was $>50 \%$ ); 3) indirectness, such as indirect population, intervention, control or outcomes; 4) imprecision manifested in wide confidence intervals; and 5) high probability of publication bias, which was downgraded once if there was evidence or high likelihood of publication bias $[14,17,18]$.

The GRADEpro GDT software (www.gradepro.org) was used to perform the "summary of findings" tables from each systematic review included.

\section{Updated meta-analysis}

\section{Risk of bias assessment}

Risk of bias was evaluated according to the PRISMA recommendations [19]. The methodological quality of studies was assessed using the Cochrane Collaboration's tool for assessing risk of bias [20]. This tool evaluates the risk of bias according to six domains: selection bias (random sequence generation and allocation concealment), performance bias (blinding of participants and personnel), detection bias (blinding of outcome assessment), attrition bias (incomplete outcome data), reporting bias (selective reporting) and other biases. In this quality assessment tool, each domain is considered as low risk, unclear risk or high risk of bias.

\section{Data synthesis}

Overview of reviews

For this section, data from the included systematic reviews with meta-analysis were extracted and presented in an "overview of reviews" table. 
Appropriate effect sizes were used, presenting RR for VAP incidence and mortality, and mean differences for duration of mechanical ventilator, ICU and/or hospital length of stay and time to VAP. When the systematic reviews with meta-analysis did not report the suitable effect size, the original data presented was used to calculate it.

\section{Updated meta-analysis}

Standardised mean differences (SMD) and 95\% confidence intervals for duration of mechanical ventilation and ICU and/or hospital length of stay, and the RR for VAP incidence and mortality were calculated between groups (intervention versus control) in each study and pooled using the Mantel-Haenszel fixed-effects model [21] or the DerSimonian-Laird random-effects model [22] depending on heterogeneity (fixed-effects model for $\mathrm{I}^{2}<50 \%$ and random-effects model for $\mathrm{I}^{2}>50 \%$ ).

Heterogeneity was assessed using the $\mathrm{I}^{2}$ statistic, and the following values were used for interpretation: low (0-40\%); moderate (30-60\%); substantial (50-90\%) and considerable (75-100\%); the corresponding p-values were also considered [14]. Sensitivity analyses were conducted by deleting each study from the model, and the pooled analyses were recalculated without each study to assess its influence on the overall SMD or RR. To test the presence of publication bias, a funnel plot and Egger's test were used [23].

In addition, an exploratory analysis was performed to analyse whether the technique used to diagnose VAP (bronchoalveolar lavage or protected specimen brushed versus endotracheal aspiration) could influence the overall effect size.

Statistical analyses were performed using STATA software (version 14; StataCorp, College Station, TX, USA).

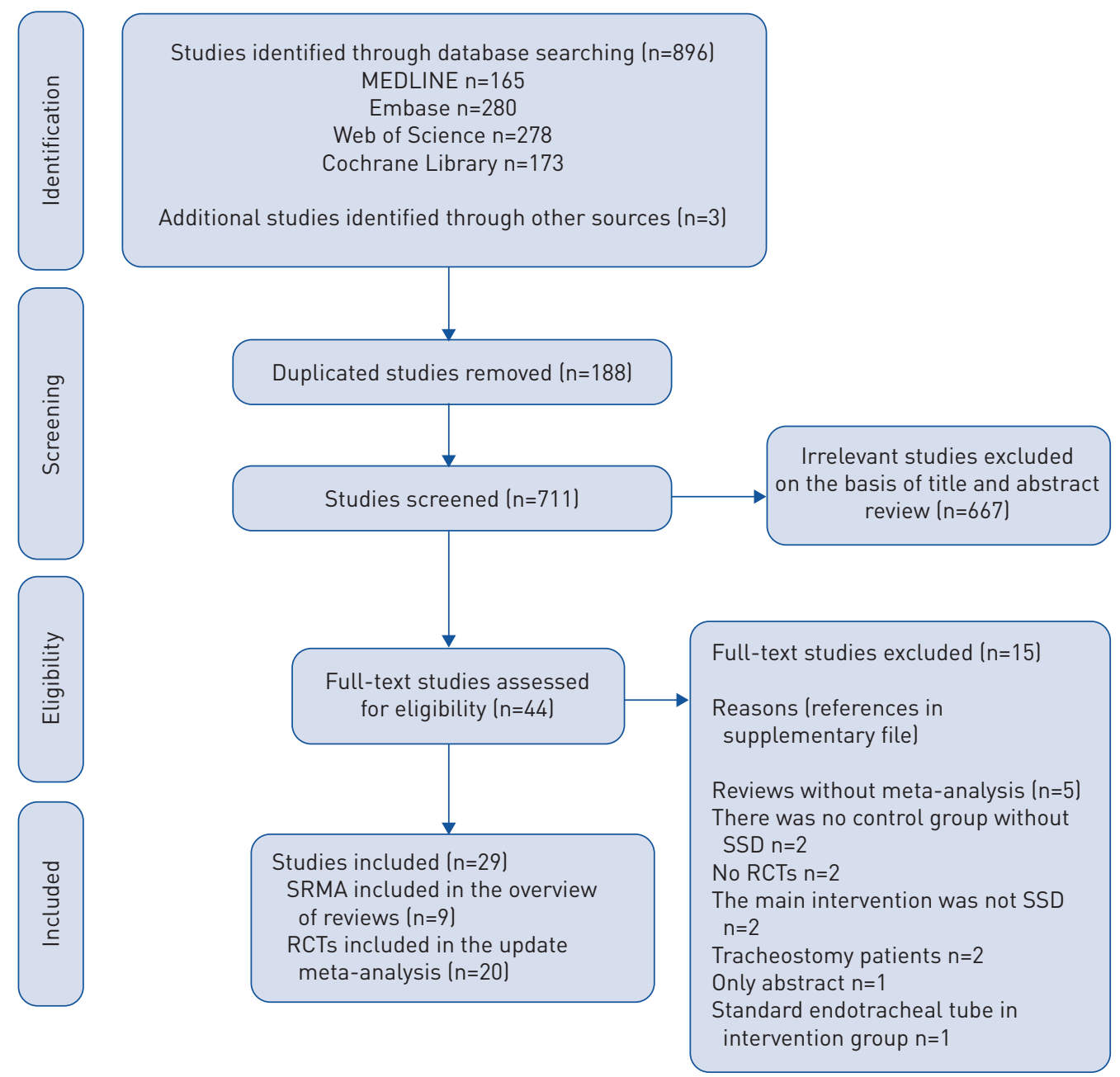

FIGURE 1 Literature search: Preferred Reporting Items for Systematic Reviews and Meta-Analyses (PRISMA) diagram. SRMA: systematic reviews with meta-analysis; RCT: randomised controlled trial; SSD: subglottic secretion drainage. 


\section{Results}

Details of the search screening process are presented in figure 1, and the reasons for excluding the 15 remaining full-text studies are shown in the supplementary material. Finally, 29 studies were included: nine systematic reviews with meta-analysis in the overview of reviews [24-32] and 20 RCTs in the updated meta-analysis [33-52].

\section{Overview of reviews}

Systematic reviews with meta-analysis characteristics

Supplementary table S1 shows the characteristics of the systematic reviews with meta-analysis included. Nine systematic reviews with meta-analysis that investigated the use of SSD for VAP prevention were included. These systematic reviews with meta-analysis were published between 2005 and 2018, and included 31876 adults across 20 unique RCTs.

Two systematic reviews with meta-analysis excluded RCTs in which, in addition to using the SSD technique, other co-interventions were carried out in the experimental group [27, 28]. One systematic review with meta-analysis excluded RCTs in which the primary outcome was not VAP micro-organisms [32].

\section{Outcome measures}

An overview of the results of the included systematic reviews is provided in supplementary table S2. All included systematic reviews with meta-analysis reported data concerning VAP incidence. The RR of the SSD intervention on the reduction of VAP incidence ranged from 0.52 (95\% CI 0.43-0.64) to 0.59 (95\% CI 0.48-0.75), showing a significant decrease in incidence in all included systematic reviews with meta-analysis.

Four systematic reviews with meta-analysis reported the RR for overall mortality $[24,26,28,30]$, three reported the RR for ICU and hospital mortality separately $[25,27,29]$, and one did not report the RR for mortality [31]; nevertheless, no review found a significant effect of SSD in reducing mortality.

The effect of SSD on the reduction of ICU and/or hospital length of stay was significant in four reviews $[24,28,29,31]$ ranging from $1.4(95 \% \mathrm{CI}-2.1--0.08)$ to $4.27(95 \% \mathrm{CI}-7.36--1.18)$ days fewer in the SSD group than in the non-SSD group.

The use of SSD shortened the duration of mechanical ventilation between 3.29 (95\% CI $-4.53--2.05)$ and 1.08 (95\% CI -2.04--0.12) days, although some reviews did not find a significant reduction in the number of mechanical ventilation days $[26,27]$.

Additionally, five systematic reviews with meta-analysis [25, 27, 28, 30, 31] reported data for the time-to-VAP outcome, showing that the SSD intervention delayed the appearance of VAP between 2.66 (95\% CI 1.06-4.26) and 4.04 (95\% CI 2.6-5.47) days.

\section{Quality assessment}

Only one review presented a rating for the certainty levels of evidence and the strength of recommendations using the GRADE tool [25].

The methodological quality and the quality of the evidence of the remaining included systematic reviews with meta-analysis were assessed. The quality of evidence for each outcome in each systematic review with meta-analysis is shown in supplementary table S3. The main limitations decreasing the level of evidence were not reporting the $\mathrm{I}^{2}$ value to assess heterogeneity or the $\mathrm{I}^{2}$ value was $>50 \%$; not assessing publication bias; and not assessing the risk of bias of the RCTs included or when $<75 \%$ of included RCTs were reported as having a low risk of bias.

The methodological quality evaluation applying the AMSTAR-2 tool showed that the main critical domains were the absence of a protocol registered before beginning the review (only one registered a previous protocol) [25]; the absence of justification for excluding studies (only three studies provided a list of excluded studies) [26, 28, 29]; and not assessing/reporting the risk of bias [27, 28] or the publication bias of the included studies $[28,32]$ (supplementary table S4).

\section{Updated meta-analysis}

\section{Studies characteristics and participants}

The 20 RCTs included were published between 1992 and 2017, and comprised 3684 adults receiving mechanical ventilation and admitted to ICUs [33-52]. The sample size of the studies ranged from 18 to 690 participants, including patients with neurological damage [35], cardiothoracic surgical patients [36, 43] and patients admitted to general and medical-surgical ICUs (supplementary table S5). 


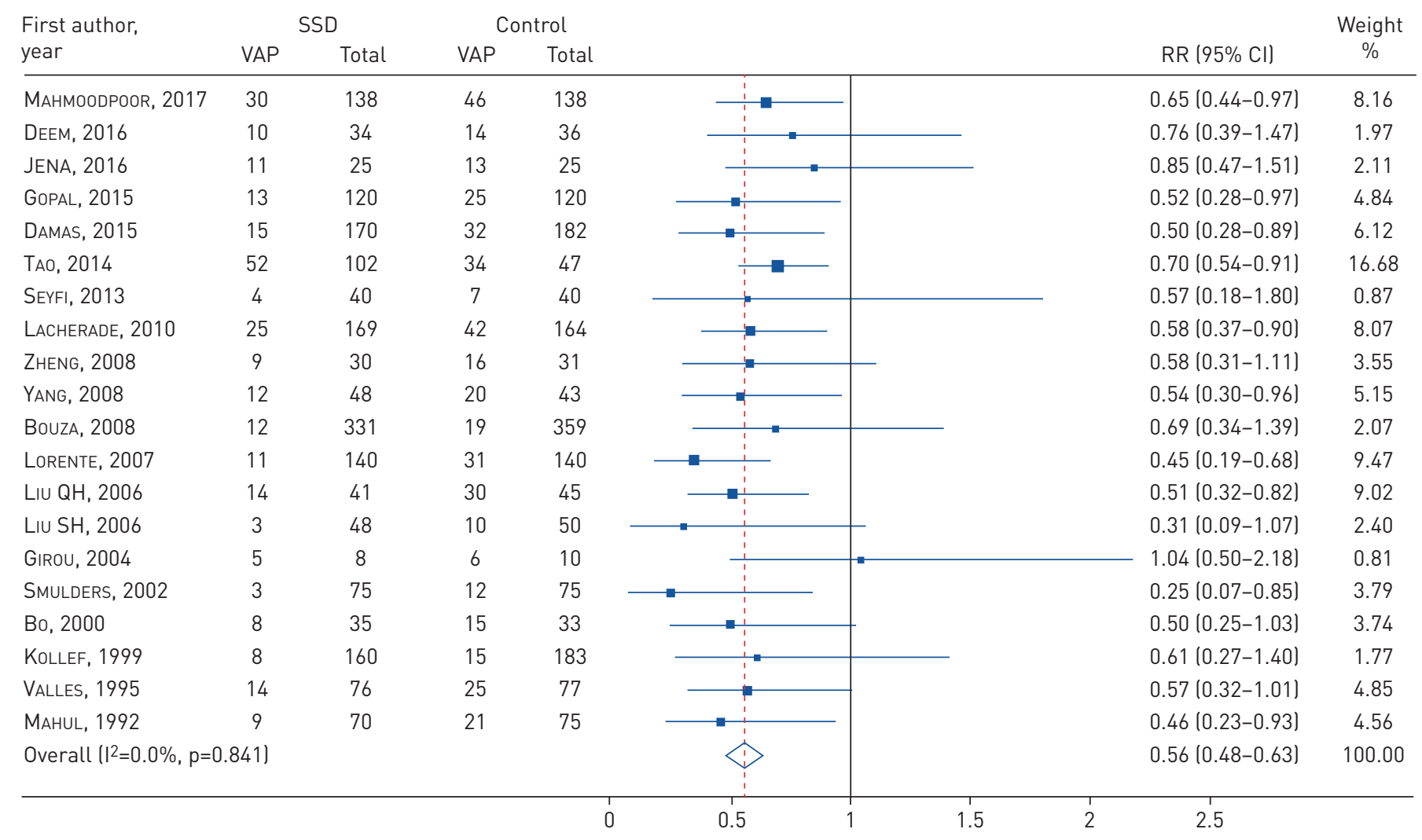

FIGURE 2 Forest plot comparing subglottic secretion drainage (SSD) versus non-SSD on the incidence of ventilator-associated pneumonia (VAP). RR: risk ratio.

\section{Pooled estimates, sensitivity analysis, publication bias VAP incidence}

All RCTs provided data for VAP incidence; SSD significantly reduced VAP incidence ( $\mathrm{R}=0.56,95 \% \mathrm{CI}$ $0.48-0.63 ; \mathrm{I}^{2}=0 \%, \mathrm{p}=0.841$ ) (figure 2 ). In the sensitivity analysis, when each study was removed, the results remained consistent across all exclusions.

\section{Secondary outcomes}

A significant difference was detected between the SSD intervention and non-SSD in mortality (RR 0.88 , 95\% CI $0.80-0.97 ; \mathrm{I}^{2}=0 \%, \mathrm{p}=0.888$ ) (figure 3). Nevertheless, there was no association found between the use of the SSD technique and the decrease in ICU length of stay (SMD $-0.12,95 \%$ CI $-0.26-0.03 ; \mathrm{I}^{2}=67.4 \%$, $\mathrm{p}=0.002$ ), hospital length of stay (SMD $-0.06,95 \% \mathrm{CI}-0.14-0.03 ; \mathrm{I}^{2}=10.6 \%, \mathrm{p}=0.348$ ) and duration of mechanical ventilation (SMD $-0.07,95 \% \mathrm{CI}-0.20-0.07 ; \mathrm{I}^{2}=62.4 \%, \mathrm{p}=0.003$ ) (supplementary figure $\mathrm{S} 1$ ).

Time-to-VAP outcomes were not pooled because the only two new RCTs included in the updated meta-analysis did not report data regarding this outcome, which does not change the data presented in the previously published meta-analysis [25].

For the mortality outcome, the sensitivity analysis showed significant reductions after excluding the studies by VAlLés et al. [51] and Liu et al. [45].

For all outcomes, both visual inspection of the funnel plot and Egger's test did not show substantial asymmetry, indicating the absence of publication bias.

The exploratory analysis performed to analyse whether the technique used to diagnose VAP (bronchoalveolar lavage or protected specimen brushed versus endotracheal aspiration) could influence the overall effect size showed that the use of SSD conveys a significant reduction in VAP in both subgroups, irrespective of the technique used to diagnose VAP.

Risk of bias assessment

Risk of bias is summarised in supplementary figure S2. 13 studies had a low risk of bias in the random sequence generation domain; however, only four had a low risk of bias in the allocation concealment item. 


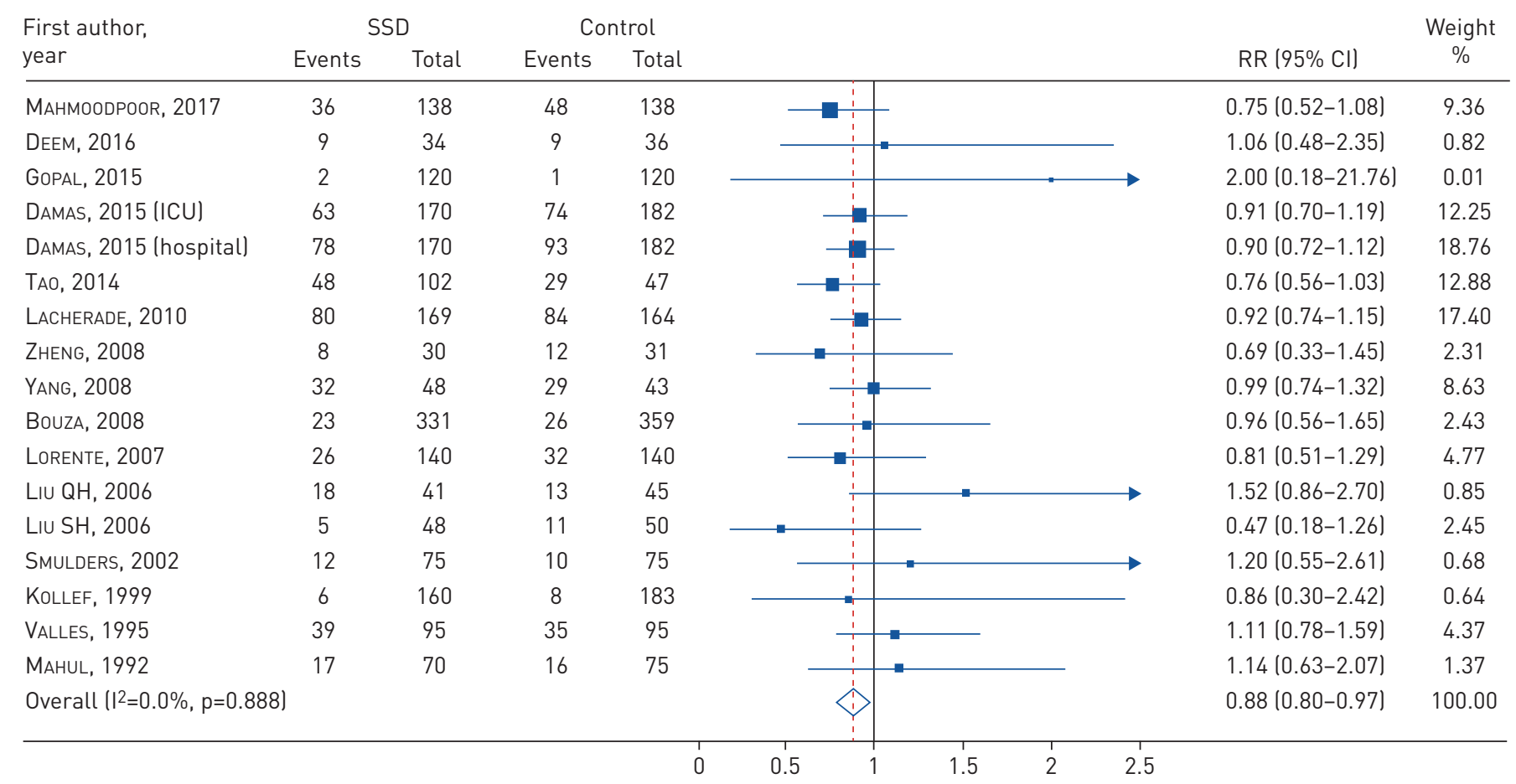

FIGURE 3 Forest plot comparing subglottic secretion drainage (SSD) versus non-SSD on mortality. RR: risk ratio; ICU: intensive care unit.

Eight studies had a low risk of bias in the blinding of personnel and outcome assessment, while four studies had a low risk of bias in all domains [33, 34, 37, 40].

\section{Discussion}

This work comprises an overview of nine systematic reviews with meta-analysis and an updated meta-analysis examining the effectiveness of SSD in reducing VAP incidence, ICU and/or hospital length of stay, duration of mechanical ventilation and mortality. SSD significantly reduced the incidence of VAP in all systematic reviews with meta-analysis and in the updated meta-analysis, but the results were not statistically significant for ICU and/or hospital length of stay and duration of mechanical ventilation. Nevertheless, contradictory results were observed regarding mortality between the overview of reviews and the updated meta-analysis results, with a significant trend towards less mortality being found in the updated meta-analysis.

One potential reason for such discordance in mortality could be a lack of power to detect significant effects, as CAROFF et al. pointed out [26, 53]. Interestingly, as sample size increases in our updated meta-analysis (3200 versus 3684), a trend towards a decrease in mortality is seen, reaching statistical significance, which may suggest a true effect. Independently, a large epidemiological study concluded that even if the strategies for VAP prevention did not decrease mortality, which could be influenced by many factors or confounders, they provided advantages to patients, their families and the healthcare system [54].

Several guidelines recommend the use of SSD as part of a care bundle to prevent VAP $[10-12,55,56]$; however, this preventive measure is not widely used. Since those patients undergoing prolonged mechanical ventilation seem to benefit more from SSD, one reason for underusing SSD (along with a higher economic cost) could be the absence of factors allowing accurate prediction of prolonged mechanical ventilation. Moreover, it has been suggested that re-intubation with SSD may represent a risk factor for VAP $[57,58]$. Regarding the cost of SSD, several cost-effectiveness studies have compared costs associated with the use of SSD endotracheal tubes versus standard endotracheal tubes among intubated patients [59-61]. Although specialised endotracheal tubes that allow SSD have a cost approximately seven times greater than conventional endotracheal tubes, all cost-effectiveness studies concluded that the SSD is a cost-effectiveness VAP preventive strategy $[60,61]$. Another reason for the underuse of SSD as a VAP preventive strategy could be that improvements in other outcomes are not observed alongside the decreased VAP rates.

Our updated meta-analysis did not find a significant reduction in the duration of mechanical ventilation associated to the use of SSD. A review addressing this issue [53] concluded that because the use of SSD 
reduces the mortality risk associated to VAP in $10 \%$ (an estimated mortality rate of 5-9\% would be reduced by $0.5-0.9 \%$ ), a sample size of tens of thousands would be necessary to reach statistical significance and thus demonstrate that SSD could reduce ICU and hospital mortality. According to this, although our updated meta-analysis provides a greater sample than those previously published, it may also be underpowered to obtain statistical significance. In addition, heterogeneity in the duration of mechanical ventilation is far greater than in mortality, thus implying that a larger sample size is needed to have enough statistical power. Nevertheless, consistent with the results found regarding mortality, the meta-analysis shows a trend towards a reduction in the duration of mechanical ventilation (along with ICU and/or hospital length of stay). In addition, other confounders such as different VAP preventive measures, treatments and patient's characteristics (comorbidities, age, main pathology, etc.) could influence this relationship.

In general, the capacity of SSD to reduce ICU and/or length of stay based on quality evidence is considered scarce. Some reviews have showed a significant decrease of ICU length of stay in the SSD group; nevertheless, these reviews had low levels of quality of evidence, which limits the reliability of the results [28-31]. In our updated meta-analysis, neither ICU nor hospital length of stay showed any improvement associated to the use of SSD.

Time to VAP was significantly increased in the SSD group in some systematic reviews; nevertheless, again, the GRADE quality of evidence was poor (low or very low for four studies $[27,28,30,31]$ and moderate for one [25]).

Our work has several limitations. First, the quality of evidence was only reported in two out of nine systematic reviews with meta-analysis. Second, the methodological quality of the systematic reviews with meta-analysis varied, ranging from critically low to high, although the majority had a low to moderate quality. Third, there was a wide heterogeneity in the population and in the type of ICUs included in the RCTs. Fourth, different diagnostic VAP criteria, such as quantitative cultures, bronchoalveolar lavage or clinical and radiologic criteria were used in the RCTs included. This could have a negative influence in terms of heterogeneity in our meta-analysis. Fifth, the use of SSD along with other VAP preventive measures did not allow us to distinguish the individual effect of SSD to prevent VAP. Moreover, these VAP bundles and/or co-interventions were different in the included studies, potentially representing a source of heterogeneity. Sixth, although a subgroup analysis by SSD techniques could be feasible in this meta-analysis, it was disregarded because recent meta-analyses [25, 62] did not report any differences between continuous and intermittent SSD to prevent VAP. Finally, due to the nature of the intervention, it could not be blinded for health staff, so only some RCTs were partially blinded.

The major strength of the present study is its development according to the Cochrane methodology and the PRISMA guidelines. Additionally, we provided a GRADE level of evidence for all included outcomes when the systematic reviews with meta-analysis did not provide a GRADE assessment. Moreover, a protocol of this overview was previously registered in PROSPERO. Finally, an updated meta-analysis including the most recent RCTs was performed, mainly yielding similar results to those obtained in previous meta-analysis but also finding a significant reduction in mortality associated to the use of SSD practice.

\section{Conclusion}

This overview provides an updated synthesis of available evidence about the use of SSD to prevent VAP, which found SSD to be an effective measure to prevent VAP and reduce mortality. However, since improvements in other outcomes such as the duration of mechanical ventilation and ICU and/or hospital length of stay were not observed alongside VAP rate improvements, further high-quality studies are necessary to elucidate the potential contribution of SSD in medical care.

Conflict of interest: None declared.

\section{References}

1 Davis KA. Ventilator-associated pneumonia: a review. J Intensive Care Med 2006; 21: 211-226.

2 Spalding MC, Cripps MW, Minshall CT. Ventilator-associated pneumonia: new definitions. Crit Care Clin 2017; 33: 277-292.

3 Melsen WG, Rovers MM, Groenwold RH, et al. Attributable mortality of ventilator-associated pneumonia: a meta-analysis of individual patient data from randomised prevention studies. Lancet Infect Dis 2013; 13: 665-671.

4 Kollef MH, Hamilton CW, Ernst FR. Economic impact of ventilator-associated pneumonia in a large matched cohort. Infect Control Hosp Epidemiol 2012; 33: 250-256.

5 Augustyn B. Ventilator-associated pneumonia: risk factors and prevention. Crit Care Nurse 2007; 27: 32-36.

6 Nseir S, Zerimech F, Jaillette E, et al. Microaspiration in intubated critically ill patients: diagnosis and prevention. Infect Disord Drug Targets 2011; 11: 413-423.

7 Mietto C, Pinciroli R, Patel N, et al. Ventilator associated pneumonia: evolving definitions and preventive strategies. Respir Care 2013; 58: 990-1007. 
8 Rouzé A, Jaillette E, Poissy J, et al. Tracheal tube design and ventilator-associated pneumonia. Respir Care 2017; 62: 1316-1323.

9 Yokoe DS, Anderson DJ, Berenholtz SM, et al. A compendium of strategies to prevent healthcare-associated infections in acute care hospitals: 2014 updates. Infect Control Hosp Epidemiol 2014; 35: 967-977.

10 Tablan OC, Anderson LJ, Besser R, et al. Guidelines for preventing health-care-associated pneumonia, 2003: recommendations of CDC and the Healthcare Infection Control Practices Advisory Committee. MMWR Recomm Rep 2004; 53: 1-36.

11 Klompas M, Branson R, Eichenwald EC, et al. Strategies to prevent ventilator-associated pneumonia in acute care hospitals: 2014 update. Infect Control Hosp Epidemiol 2014; 35: 915-936.

12 Álvarez-Lerma F, Palomar-Martínez M, Sánchez-García M, et al. Prevention of ventilator-associated pneumonia: the multimodal approach of the Spanish ICU "Pneumonia Zero" program. Crit Care Med 2018; 46: 181-188.

13 Moher D, Liberati A, Tetzlaff J, et al. Reprint - preferred reporting items for systematic reviews and meta-analyses: the PRISMA statement. Phys Ther 2009; 89: 873-880.

14 Higgins JP, Green S. Cochrane Handbook for Systematic Reviews of Interventions Version 5.1.0. Cochrane Collaboration 2011 http://handbook-5-1.cochrane.org/

15 Shea BJ, Reeves BC, Wells G, et al. AMSTAR 2: a critical appraisal tool for systematic reviews that include randomised or non-randomised studies of healthcare interventions, or both. BMJ 2017; 358: j4008.

16 Balshem $\mathrm{H}$, Helfand M, Schünemann HJ, et al. GRADE guidelines: 3. Rating the quality of evidence. J Clin Epidemiol 2011; 64: 401-406.

17 Guyatt GH, Oxman AD, Vist GE, et al. GRADE: an emerging consensus on rating quality of evidence and strength of recommendations. BMJ 2008; 336: 924-926.

18 Schünemann H BJ, Guyatt G, Oxman A, editors. GRADE Handbook for Grading Quality of Evidence and Strength of Recommendations. The GRADE Working Group, 2013. www.guidelinedevelopment.org/handbook Date last updated: October 2013.

19 Moher D, Liberati A, Tetzlaff J, et al. Preferred reporting items for systematic reviews and meta-analyses: the PRISMA statement. J Clin Epidemiol 2009; 62: 1006-1012.

20 Higgins JP, Altman DG, Gøtzsche PC, et al. The Cochrane Collaboration's tool for assessing risk of bias in randomised trials. BMJ 2011; 343: d5928.

21 Mantel N, Haenszel W. Statistical aspects of the analysis of data from retrospective studies of disease. $J$ Nat Cancer Inst 1959; 22: 719-748.

22 DerSimonian R, Laird N. Meta-analysis in clinical trials. Control Clin Trials 1986; 7: 177-188.

23 Egger M, Davey Smith G, Schneider M, et al. Bias in meta-analysis detected by a simple, graphical test. BMJ 1997; 315: 629-634.

24 Sun X, Zhang J, Jiang T, et al. [Comprehensive effect of subglottic secretion drainage on patients with mechanical ventilation in ICU: a meta-analysis]. Zhonghua Wei Zhong Bing Ji Jiu Yi Xue 2017; 29: 586-591.

25 Mao Z, Gao L, Wang G, et al. Subglottic secretion suction for preventing ventilator-associated pneumonia: an updated meta-analysis and trial sequential analysis. Crit Care 2016; 20: 353.

26 Caroff DA, Li L, Muscedere J, et al. Subglottic secretion drainage and objective outcomes: a systematic review and meta-analysis. Crit Care Med 2016; 44: 830-840.

27 Frost SA, Azeem A, Alexandrou E, et al. Subglottic secretion drainage for preventing ventilator associated pneumonia: a meta-analysis. Aust Crit Care 2013; 26: 180-188.

28 Leasure AR, Stirlen J, Lu SH. Prevention of ventilator-associated pneumonia through aspiration of subglottic secretions: a systematic review and meta-analysis. Dimens Crit Care Nurs 2012; 31: 102-117.

29 Muscedere J, Rewa O, McKechnie K, et al. Subglottic secretion drainage for the prevention of ventilator-associated pneumonia: a systematic review and meta-analysis. Crit Care Med 2011; 39: 1985-1991.

30 Wang F, Bo L, Tang L, et al. Subglottic secretion drainage for preventing ventilator-associated pneumonia: an updated meta-analysis of randomized controlled trials. J Trauma Acute Care Surg 2012; 72: 1276-1285.

31 Dezfulian C, Shojania K, Collard HR, et al. Subglottic secretion drainage for preventing ventilator-associated pneumonia: a meta-analysis. Am J Med 2005; 118: 11-18.

32 Huang XA, Du YP, Fu BB, et al. Influence of subglottic secretion drainage on the microorganisms of ventilator associated pneumonia: a meta-analysis for subglottic secretion drainage. Medicine 2018; 97: e11223.

33 Mahmoodpoor A, Hamishehkar H, Hamidi M, et al. A prospective randomized trial of tapered-cuff endotracheal tubes with intermittent subglottic suctioning in preventing ventilator-associated pneumonia in critically ill patients. J Crit Care 2017; 38: 152-156.

34 Deem S, Yanez D, Sissons-Ross L, et al. Randomized pilot trial of two modified endotracheal tubes to prevent ventilator-associated pneumonia. Ann Am Thorac Soc 2016; 13: 72-80.

35 Jena S, Kamath S, Masapu D, et al. Comparison of suction above cuff and standard endotracheal tubes in neurological patients for the incidence of ventilator-associated pneumonia and in-hospital outcome: a randomized controlled pilot study. Indian J Crit Care Med 2016; 20: 261-266.

36 Gopal S, Luckraz H, Giri R, et al. Significant reduction in ventilator-associated pneumonia with the Venner-PneuX system in high-risk patients undergoing cardiac surgery: the Low Ventilator-Associated-Pneumonia study. Eur J Cardiothorac Surg 2015; 47: e92-e96.

37 Damas P, Frippiat F, Ancion A, et al. Prevention of ventilator-associated pneumonia and ventilator-associated conditions: a randomized controlled trial with subglottic secretion suctioning. Crit Care Med 2015; 43: 22-30.

38 Tao Z, Zhao S, Yang G, et al. [Effect of two methods of subglottic secretion drainage on the incidence of ventilator-associated pneumonia]. Zhonghua Jie He He Hu Xi Za Zhi 2014; 37: 283-286.

39 Seyfi S, Latifi S, Shirkhani Z. Effect of subglottic secretion drainage on the ventilator-associated pneumonia in ICU patients. J Babol Univ Med Sci 2013; 15: 58-62.

40 Lacherade JC, De Jonghe B, Guezennec $\mathrm{P}$, et al. Intermittent subglottic secretion drainage and ventilator-associated pneumonia: a multicenter trial. Am J Respir Crit Care Med 2010; 182: 910-917.

41 Zheng RQ, Lin H, Shao J, et al. [A clinical study of subglottic secretion drainage for prevention of ventilation associated pneumonia]. Zhongguo Wei Zhong Bing Ji Jiu Yi Xue 2008; 20: 338-340. 
42 Yang CS, Qiu HB, Zhu YP, et al. [Effect of continuous aspiration of subglottic secretions on the prevention of ventilator-associated pneumonia in mechanically ventilated patients: a prospective, randomized, controlled clinical trial]. Zhonghua Nei Ke Za Zhi 2008; 47: 625-629.

43 Bouza E, Pérez MJ, Muñoz P, et al. Continuous aspiration of subglottic secretions in the prevention of ventilator-associated pneumonia in the postoperative period of major heart surgery. Chest 2008; 134: 938-946.

44 Lorente L, Lecuona M, Jiménez A, et al. Influence of an endotracheal tube with polyurethane cuff and subglottic secretion drainage on pneumonia. Am J Respir Crit Care Med 2007; 176: 1079-1083.

45 Liu QH, He LX, Hu BJ, et al. [Comprehensive prevention and pathogenesis of ventilator-associated pneumonia in elderly patients: a prospective, randomized, case-control clinical trial]. Zhonghua Nei Ke Za Zhi 2006; 45: 717-720.

46 Liu SH, Yan XX, Cao SQ, et al. [The effect of subglottic secretion drainage on prevention of ventilator-associated lower airway infection]. Zhonghua Jie He He Hu Xi Za Zhi 2006; 29: 19-22.

47 Girou E, Buu-Hoi A, Stephan F, et al. Airway colonisation in long-term mechanically ventilated patients. Effect of semi-recumbent position and continuous subglottic suctioning. Intensive Care Med 2004; 30: 225-233.

48 Smulders $\mathrm{K}$, van der Hoeven $\mathrm{H}$, Weers-Pothoff $\mathrm{I}$, et al. A randomized clinical trial of intermittent subglottic secretion drainage in patients receiving mechanical ventilation. Chest 2002; 121: 858-862.

49 Bo H, He L, Qu J. [Influence of the subglottic secretion drainage on the morbidity of ventilator associated pneumonia in mechanically ventilated patients]. Zhonghua Jie He He Hu Xi Za Zhi 2000; 23: 472-474.

50 Kollef MH, Skubas NJ, Sundt TM. A randomized clinical trial of continuous aspiration of subglottic secretions in cardiac surgery patients. Chest 1999; 116: 1339-1346.

51 Vallés J, Artigas A, Rello J, et al. Continuous aspiration of subglottic secretions in preventing ventilator-associated pneumonia. Ann Intern Med 1995; 122: 179-186.

52 Mahul P, Auboyer C, Jospe R, et al. Prevention of nosocomial pneumonia in intubated patients: respective role of mechanical subglottic secretions drainage and stress ulcer prophylaxis. Intensive Care Med 1992; 18: 20-25.

53 Lacherade J-C, Azais M-A, Pouplet C, et al. Subglottic secretion drainage for ventilator-associated pneumonia prevention: an underused efficient measure. Ann Transl Med 2018; 6: 422.

54 Rello J, Ollendorf DA, Oster G, et al. Epidemiology and outcomes of ventilator-associated pneumonia in a large US database. Chest 2002; 122: 2115-2121.

55 SARI Working Group. Guidelines for the Prevention of Ventilator-Associated Pneumonia in Adults in Ireland. Dublin, HSE Health Protection Surveillance Centre (HPSC), 2011.

56 American Thoracic Society, Infectious Diseases Society of America. Guidelines for the management of adults with hospital-acquired, ventilator-associated, and healthcare-associated pneumonia. Am J Respir Crit Care Med 2005; 171: 388-416.

57 Masjedi M, Zand F, Sabetian G, et al. Early replacement of conventional endotracheal tube with endotracheal tube with subglottic suction port for the new intensive care patients; preventive or problematic against ventilator associated events? J Pure Appl Microbio 2016; 10: 2655-2662.

58 Doyle A, Fletcher A, Carter J, et al. The incidence of ventilator-associated pneumonia using the PneuX System with or without elective endotracheal tube exchange: a pilot study. BMC Res Notes 2011; 4: 92.

59 Kelley SD. Number needed to treat for subglottic secretion drainage technology as a ventilator-associated pneumonia prevention strategy. Crit Care 2012; 16: 446.

60 Speroni KG, Lucas J, Dugan L, et al. Comparative effectiveness of standard endotracheal tubes vs. endotracheal tubes with continuous subglottic suctioning on ventilator-associated pneumonia rates. Nurs Econ 2011; 29: 15-20.

61 Hallais C, Merle V, Guitard PG, et al. Is continuous subglottic suctioning cost-effective for the prevention of ventilator-associated pneumonia? Infect Control Hosp Epidemiol 2011; 32: 131-135.

62 Wen Z, Zhang H, Ding J, et al. Continuous versus intermittent subglottic secretion drainage to prevent ventilator-associated pneumonia: a systematic review. Crit Care Nurse 2017; 37: e10-e17. 